\title{
Micellar emulsions composed of mPEG-PCL/MCT as novel nanocarriers for systemic delivery of genistein: a comparative study with micelles
}

This article was published in the following Dove Press journal:

International Journal of Nanomedicine

I October 2015

Number of times this article has been viewed

\author{
Tianpeng Zhang* \\ Huan Wang* \\ Yanghuan Ye \\ Xingwang Zhang \\ Baojian Wu
}

Division of Pharmaceutics, College of Pharmacy, Jinan University, Guangzhou, People's Republic of China

*These authors contributed equally to this work
Correspondence: Xingwang Zhang; Baojian Wu

Division of Pharmaceutics, College of Pharmacy, Jinan University, 60I West Huangpu Avenue, Guangzhou 510632, People's Republic of China

Tel +862085220482

Email pharmazxw@gmail.com; bj.wu@ hotmail.com

\begin{abstract}
Polymeric micelles receive considerable attention as drug delivery vehicles, depending on the versatility in drug solubilization and targeting therapy. However, their use invariably suffers with poor stability both in in vitro and in vivo conditions. Here, we aimed to develop a novel nanocarrier (micellar emulsions, MEs) for a systemic delivery of genistein (Gen), a poorly soluble anticancer agent. Gen-loaded MEs (Gen-MEs) were prepared from methoxy poly(ethylene glycol)-block-( $\varepsilon$-caprolactone) and medium-chain triglycerides (MCT) by solventdiffusion technique. Nanocarriers were characterized by dynamic light scattering, transmission electron microscopy, and in vitro release. The resulting Gen-MEs were approximately $46 \mathrm{~nm}$ in particle size with a narrow distribution. Gen-MEs produced a different in vitro release profile from the counterpart of Gen-ME. The incorporation of MCT significantly enhanced the stability of nanoparticles against dilution with simulated body fluid. Pharmacokinetic study revealed that MEs could notably extend the mean retention time of Gen, 1.57- and 7.38-fold as long as that of micelles and solution formulation, respectively, following intravenous injection. Furthermore, MEs markedly increased the elimination half-life $\left(t_{1 / 2 \beta}\right)$ of Gen, which was 2.63-fold larger than that of Gen solution. Interestingly, Gen distribution in the liver and kidney for MEs group was significantly low relative to the micelle group in the first 2 hours, indicating less perfusion in such two tissues, which well accorded with the elongated mean retention time. Our findings suggested that MEs may be promising carriers as an alternative of micelles to systemically deliver poorly soluble drugs.
\end{abstract}

Keywords: genistein, micellar emulsions, stability, pharmacokinetics, tissue distribution

\section{Introduction}

Genistein (Gen), 4',5,7-trihydroxyisoflavone (Figure 1), is a highly active compound found in soybean, and has gotten close attention recently due to potent bioactivities. ${ }^{1-3}$ It is shown that Gen has good potential in anti-oxidation, antiproliferation, and antitumor. Accordingly, it is highly preferred to use for prevention and treatment of cardiovascular diseases, ${ }^{4}$ osteoporosis,${ }^{5}$ and various cancers, such as breast, prostate, skin, and ovarian cancer. ${ }^{6-8}$ The underlying mechanisms involve inhibition of protein tyrosine kinases and NF- $\kappa \mathrm{B}$, induction of apoptosis, downregulation of genes associated with cell proliferation, etc. ${ }^{9-11}$ However, the therapeutic benefits of Gen remain challenging owing to its poor solubility $(\sim 1.43 \mu \mathrm{g} / \mathrm{mL})^{12}$ and low bioavailability. There is an intrinsic demand for developing a suitable formulation to address the systemic delivery of Gen.

To enable and improve the systemic delivery of poorly soluble drugs, various nanotechnology-based drug delivery systems have been explored. These promising 


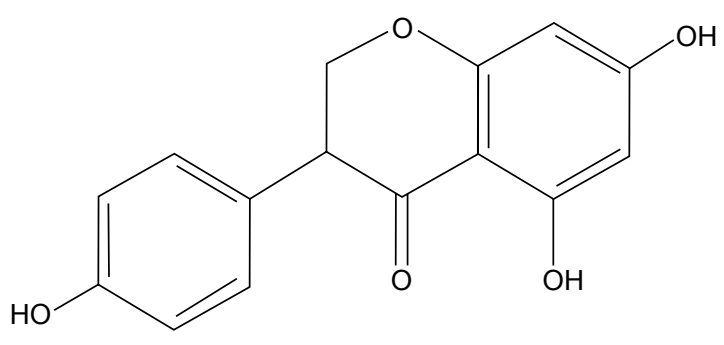

Figure I Chemical structure of genistein.

carriers include liposomes, ${ }^{13}$ micelles, ${ }^{14}$ nanoemulsions, ${ }^{15}$ lipid nanoparticles, ${ }^{16,17}$ and polymeric nanoparticles. ${ }^{18,19}$ Among these, micelles have been extensively investigated for smart delivery of poorly water-soluble or insoluble drugs, especially polymeric micelles. ${ }^{20}$ Polymeric micelles are assembled by amphiphilic block copolymer that have a hydrophobic core and a hydrophilic shell in structure. The core serves as a reservoir to solubilize and load drug, whereas the shell has the ability to increase the stability of colloidal particles. Micelles with a suitable PEG chain can effectively avoid phagocytosis by the reticuloendothelial system (RES) following opsonization by complement proteins, thus prolonging the body circulation time of nanoparticles. ${ }^{21}$ However, micellar carriers are accompanied by some shortcomings, including drug expulsion from the carrier, dissociation upon dilution by the blood stream, and accelerated blood clearance phenomenon. ${ }^{22}$ To ameliorate the drug loading and biofate of micelles, it is workable to introduce a lipophilic component into the micellar system whereby to intensify the core stability and reduce the drug expulsion. The participation of lipophilic component can create a new nanostructure that is neither different from micelle nor from emulsions. This new nanostructure can be deemed as micellar emulsions (MEs), as illustrated in Figure 2, which may be potential as drug delivery system. Till date, MEs have not been investigated for systemic delivery of poorly soluble drugs as an alternative of micelles.
In this work, methoxy poly(ethylene glycol)-block-( $\varepsilon$ caprolactone) (mPEG-PCL) and medium-chain triglycerides (MCTs) were used to prepare MEs, aiming to improve the Gen loading and achieve a good systemic delivery. The performance of MEs and suitability as injectable nanocarriers was evaluated by in vitro release, physiological stability, pharmacokinetics, and tissue distribution with Gen-loaded micelles (Gen-MEs) as reference.

\section{Materials and methods}

\section{Materials}

Genistein and Tween 80 were purchased from Aladdin Chemicals (Shanghai, People's Republic of China). MCT was kindly gifted by Gattefosse (Shanghai, People's Republic of China). The copolymer of mPEG-PCL was synthesized in our laboratory as described. ${ }^{23}$ Deionized water was prepared by a water purification system (Sichuan Wortel Water Treatment Equipment Co., Ltd., Chengdu, People's Republic of China). All other chemicals were of analytical grade and used as received.

\section{Preparation of Gen-loaded micellar emulsions}

Gen-loaded micellar emulsions (Gen-MEs) were prepared by the solvent-diffusion technique as previously described with minor modification. ${ }^{24}$ Briefly, Gen, MCT, and mPEG-PCL at the ratio of $4 / 5 / 50(\mathrm{w} / \mathrm{w})$ were dissolved in hot absolute ethanol, and then rapidly injected into deionized water (1:5, $\mathrm{v} / \mathrm{v})$ under agitation. Self-assembly occurred upon the solvent diffusion into the aqueous phase, resulting in formation of Gen-MEs. Afterward, the organic solvent was removed from the system under reduced pressure by a rotatory evaporator (Rotavapor R-210, Buchi, Uster, Switzerland). Finally, Gen-MEs were appropriately condensed to reach a Gen concentration of $4 \mathrm{mg} / \mathrm{mL}$. Factors affecting the performance of Gen-MEs were screened, including the ratios of $\mathrm{MCT} / \mathrm{mPEG}-\mathrm{PCL}$, drug/mPEG-PCL, and organic/aqueous
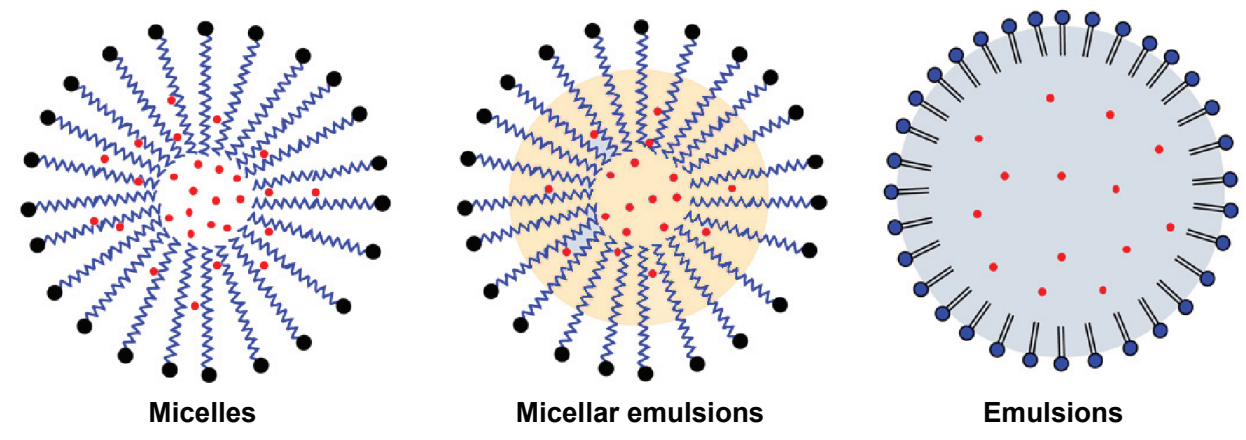

Figure 2 Schematic illustration of structures of micelles, micellar emulsions, and emulsions. 
phase volume. Gen-micelles, as a reference preparation, were prepared following the same procedure.

\section{Characterization of Gen-MEs}

The particle size and $\zeta$ potential of MEs were measured by Zetasizer Nano ZS (Malvern Instruments, Malvern, UK). To measure the particle size and zeta potential, $50 \mu \mathrm{L}$ of Gen1-MEs sample was diluted with deionized water to $1 \mathrm{~mL}$, and then subjected to laser diffraction after equilibration for 2 minutes at $25^{\circ} \mathrm{C}$. The data were processed using the build-in software based on the output of particle size and $\zeta$ potential.

The morphology of Gen-MEs was examined with transmission electron microscopy (TEM, JEM-1230, JEOL, Tokyo, Japan). Briefly, an aliquot of Gen-MEs was placed on a carbon-coated copper grid and allowed to dry at room temperature. The grid loading Gen-MEs particles were then subjected to TEM inspection, and micrographs pertaining to nanoparticles were taken at an acceleration voltage of $100 \mathrm{kV}$.

For measurement of entrapment efficiency (EE) and drug load (DL), the free Gen in MEs system was separated by ultrafiltration centrifugation with Amicon ${ }^{\circledR}$ Ultra- 0.5 centrifugal filter (molecular weight cut-off [MWCO] $50 \mathrm{kDa}$, EMD Millipore, Billerica, MA, USA). Briefly, $400 \mu \mathrm{L}$ of sample was transferred to a centrifugal filtration tube and centrifuged at $10,000 \times g$ for 10 minutes. The Gen concentration in the filtrate was quantified by established high performance liquid chromatography (HPLC) method below. To quantify the total drug, Gen-MEs were dissolved with methanol, and Gen concentration was analyzed by HPLC after suitable dilution. EE was defined as the percentage of Gen encapsulated in MEs to the total Gen in the formulation, and calculated by the equation of $\mathrm{EE}(\%)=\left(1-\mathrm{C}_{\mathrm{fre}} / \mathrm{C}_{\text {tot }}\right) \times 100 \%$. DL could be estimated from the equation of DD $(\%)=M_{d r u} \cdot E E /\left(M_{d r u}+M_{e x c}\right) \times 100 \%$. $\mathrm{C}_{\text {fre }}$ and $\mathrm{C}_{\text {tot }}$ were respectively the free and total Gen concentration in the MEs quantified based on HPLC; $M_{d r u}$ and $M_{\text {exc }}$ denoted the amount of drug and all excipients used in the formulation quantified based on weighing.

\section{In vitro release study}

The in vitro release of Gen from MEs was performed using a dialysis bag (MWCO 8,000-14,000 Da). To mimic the release behavior of MEs in the physiological conditions, simulated body fluid (SBF) was adopted as the release medium and prepared as described previously. ${ }^{25}$ In brief, $2 \mathrm{~mL}$ of Gen-MEs or Gen-micelles was dialyzed against $900 \mathrm{~mL}$ of SBF (pH 7.4) containing 2\% (w/v) Tween 80 at $37^{\circ} \mathrm{C}$ under stirring. At predetermined time points, $500 \mu \mathrm{L}$ of sample was withdrawn and immediately replenished with the same volume of fresh medium. Gen concentration in the external medium was determined by HPLC, and the percentage of drug release was calculated as mean $\pm \mathrm{SD}$ $(n=3)$.

\section{Stability study}

The physical stability of Gen-MEs and Gen-micelles was investigated upon storage at $25^{\circ} \mathrm{C}$ and $4^{\circ} \mathrm{C}$ for 1 week. During the storage period, the particle size as well as its distribution was monitored by Zetasizer Nano ZS (Malvern Instruments, Malvern UK). Moreover, the physiological stability of GenMEs and Gen-micelles in the blood was evaluated in vitro using SBF. Briefly, $2 \mathrm{~mL}$ of preparations loading Gen was added into $200 \mathrm{~mL}$ of SBF and maintained at $37^{\circ} \mathrm{C}$ with stirring. The particle size was measured in real time upon circulation.

\section{Quantification of genistein}

Genistein concentration in the in vitro samples was determined by HPLC. Instrument was the same as the one used in our previous report. ${ }^{26}$ Gen was eluted by a Syncronis C18 column $(5 \mu \mathrm{m}, 4.6 \mathrm{~mm} \times 250 \mathrm{~mm}$, Thermo Fisher Scientific, Waltham, MA, USA) guarded with a precolumn at $40^{\circ} \mathrm{C}$ and detected at $262 \mathrm{~nm}$. Twenty microliters of sample was injected into the HPLC system running at a flow rate of $1.0 \mathrm{~mL} / \mathrm{min}$. The mobile phase was composed of $70 \%$ methanol and $30 \%$ water with $0.1 \%$ phosphoric acid.

The in vivo samples were quantified by UPLC-QTOF/ MS (ACQUITY UPLC, Waters, Milford, MA, USA). Instrument configuration and parameter settings referred to the previous publication. ${ }^{27}$ Chromatographic separation was performed on an ACQUITY UPLC BEH column $(2.1 \times 50 \mathrm{~mm}$, $1.7 \mu \mathrm{m})$. A gradient elution was applied using formic acid in water $(0.1 \%$, mobile phase $A)$ vs formic acid in acetonitrile $(0.1 \%$, mobile phase B) at a flow rate of $0.45 \mathrm{~mL} / \mathrm{min}$. The gradient elution program was $5 \% \mathrm{~B}$ at $0-1$ minutes, $5 \%-95 \% \mathrm{~B}$ at $1-3$ minutes, $95 \% \mathrm{~B}$ at $3-3.5$ minutes, and 95\%-5\% B at 3.5-4 minutes. Quantitation was performed based on the full scan analysis and extracted ion chromatograms using MassLynx version 4.1. A typical chromatogram for Gen analysis was shown in Figure 3A with drug elution at 2.72 minutes, and the extracted ion chromatogram was included in Figure 3B.

\section{Pharmacokinetic study}

Pharmacokinetic study was performed in Sprague-Dawley rats (male, 220-250 g). The animal experiment protocol was 


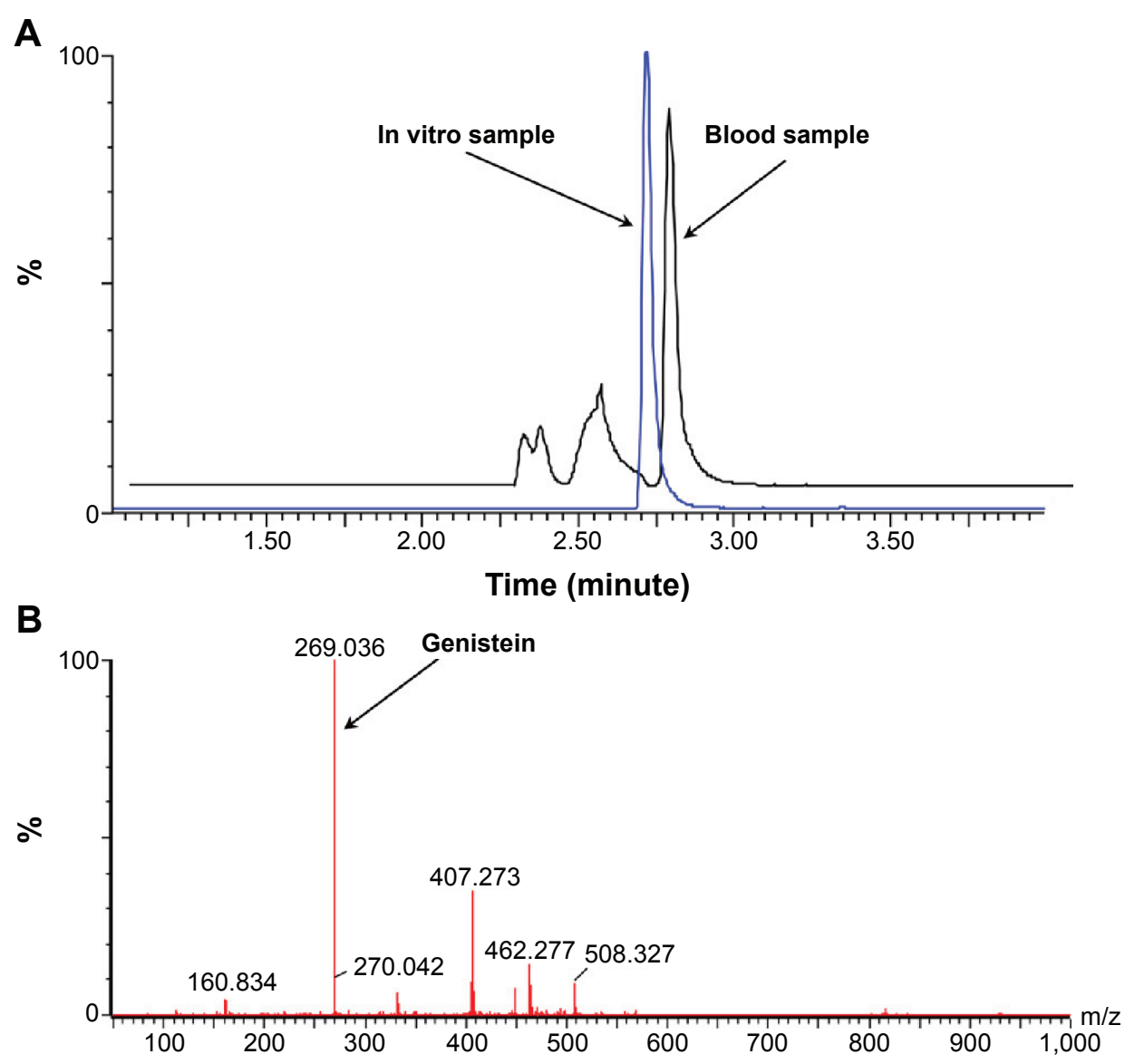

Figure 3 Quantification of Gen by UPLC-QTOF/MS.

Notes: (A) Representative chromatograms for quantification of Gen by UPLC-QTOF/MS. (B) The extracted ion chromatogram of Gen in plasma.

Abbreviations: Gen, genistein; MS, mass spectroscopy; QTOF, quadrupole time of flight; UPLC, ultra performance liquid chromatography.

reviewed and approved by the Experimental Animal Ethical Committee of Jinan University (Guangzhou, People's Republic of China). The rats were fasted overnight prior to administration but allowed free access to water. Experimental rats were randomly divided into three groups $(n=5)$, namely Gen-MEs, Gen-micelles, and Gen solution group, where a co-solvent of water/alcohol was used to prepare Gen solution formulation. The rats were administrated through intrajugular injection with a dose of $10 \mathrm{mg} / \mathrm{kg}$. At 0.083 , $0.25,0.5,1,2,4,6,8,12$, and 24 hours after administration, $0.25 \mathrm{~mL}$ of blood was collected into heparinized tubes via the jugular vein and centrifuged at $5,000 \times g$ for 8 minutes to collect the plasma.

A deproteinization procedure was employed to extract Gen from the plasma prior to analysis. Briefly, $100 \mu \mathrm{L}$ of plasma sample was mixed with $400 \mu \mathrm{L}$ of acetonitrile containing $1 \mu \mathrm{M}$ SNX-2112 (internal standard) and vortexed vigorously for 3 minutes. After centrifugation at $13,000 \times g$ for 10 minutes, the supernatants were transferred into clean tubes and evaporated with Concentrator Plus (Eppendorf,
Hamburg, Germany) at $30^{\circ} \mathrm{C}$. The residues were reconstituted in $200 \mu \mathrm{L}$ of $50 \%$ acetonitrile and then subjected to UPLC-QTOF/MS analysis after centrifugation.

\section{Tissue distribution}

Tissue distribution of Gen after dosing was determined in $\mathrm{SD}$ rats. The rats were randomly divided into two groups $(\mathrm{n}=5)$ and intravenously administrated with Gen-MEs or Gen-micelles, respectively, at the dose of $10 \mathrm{mg} / \mathrm{kg}$. At 2, 10, and 24 hours after injection, the rats were sacrificed by cervical dislocation, and the organs of heart, liver, spleen, lung, kidney, and intestine were harvested. The tissue samples were washed with ice-cold saline and weighted after cleaning with filter paper.

The tissues were homogenized with saline solution at the ratio of 1:2 (w/v) under ice bath condition. Then, $200 \mu \mathrm{L}$ of homogenate was mixed with $800 \mu \mathrm{L}$ of acetonitrile containing $0.5 \mu \mathrm{M}$ SNX-2112. The mixtures were vortexed and centrifuged at $13,000 \times g$ for 10 minutes at $4^{\circ} \mathrm{C}$. The supernatants were collected and dried under vacuum using 
Concentrator Plus at $30^{\circ} \mathrm{C}$. The dried residues were reconstituted into $200 \mu \mathrm{L}$ of $50 \%$ acetonitrile. Gen in the extracted residuals was quantified by UPLC-QTOF/MS.

\section{Results and discussion Characterization of Gen-MEs}

The solvent-diffusion technique was frequently used to prepare drug-loaded micelles or nanoparticles. In the preliminary experiment, it was found that the ratios of Gen/ mPEG-PCL, MCT/mPEG-PCL, and aqueous/organic phase upon preparation impacted the formulation properties of MEs. In order to gain a suitable formulation, we investigated the effect of those factors on the particle size and distribution of Gen-MEs by varying the level of one factor but keeping others unchanged. The effects of formulation variables on the formulation properties are shown in Figure 4.

The ratio of MCT/mPEG-PCL had little effect on the particle size of MEs (Figure 4A). High ratio of MCT resulted in the increase of MEs' particle size and wide distribution. MCT can entrap into the hydrophobic segment of mPEG-PCL, expand the core volume, and affect the self-assembly process, thereby raising the diameter of MEs when more MCT used. Nevertheless, the increase in particle size was not sharp along the gradient, indicating that the participation of MCT would not cause significant change of colloidal structure. Considering the advantage of small particle size in retarding phagocytosis, a low ratio of MCT/mPEG/PCL (1:10) was selected. Likewise, the amount of drug affected the performance of MEs that a high proportion of Gen would significantly increase the particle size of MEs (Figure 4B), other than the drug load. The drug load increased with the increase of drug amount below 1:12.5. Furthermore, the drug load was significantly greater than that of micelles group (no MCT), indicating an appropriate amount of MCT can enhance the drug load by solubilization. Another factor affecting the properties of MEs was the volume ratio of organic phase vs aqueous phase upon preparation (Figure 4C). At a high organic/aqueous phase ratio, the formed MEs were relatively
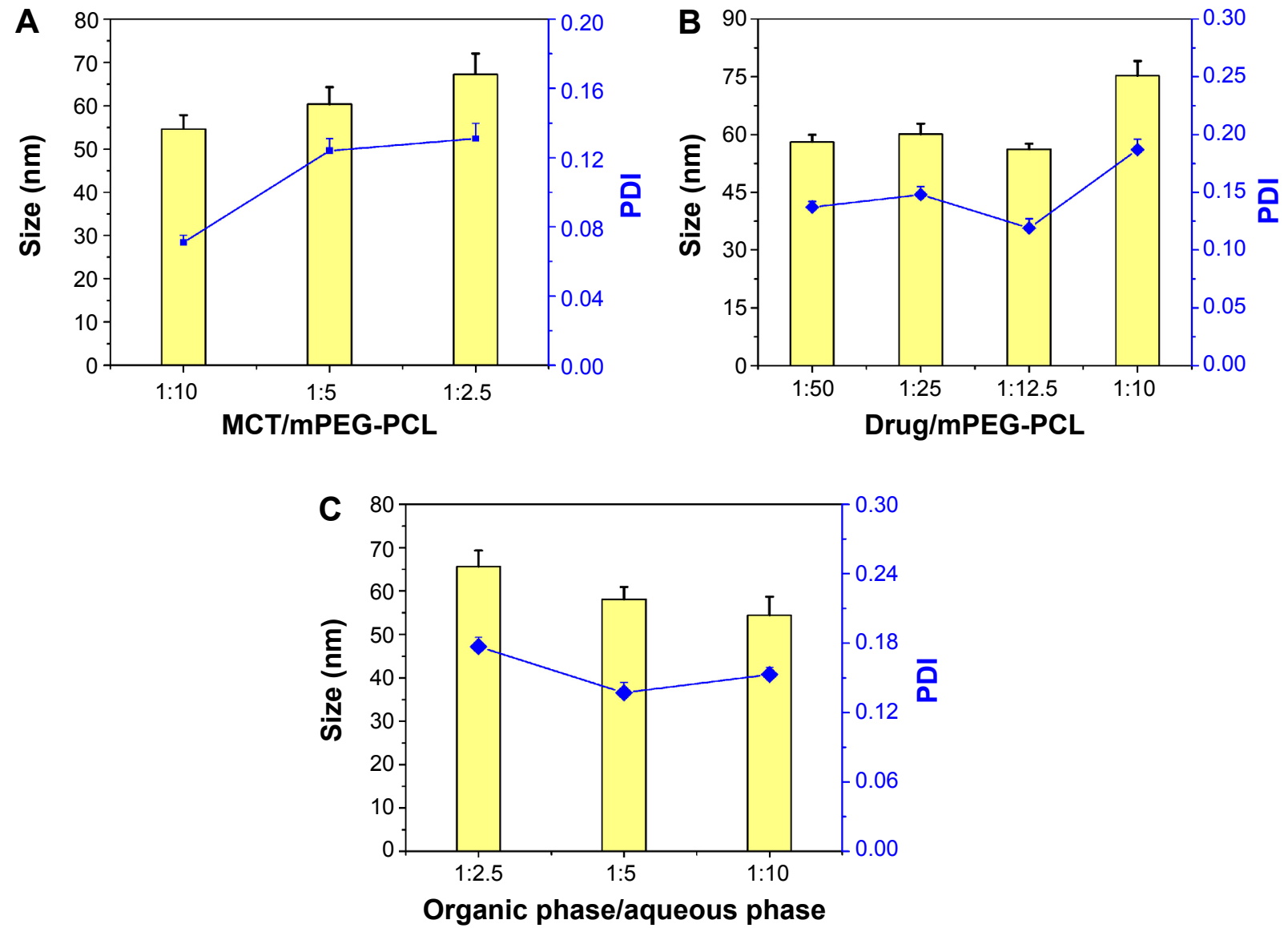

Figure 4 Formulation screening of Gen-MEs

Notes: The effects of MCT/mPEG-PCL ratio (A), drug/mPEG-PCL ratio (B), and organic/aqueous phase volume ratio upon injection (C) on particle size as well as PDI of Gen-MEs ( $n=3$, mean $\pm S D$ ).

Abbreviations: Gen-MEs, genistein-loaded micellar emulsions; MCT, medium-chain triglycerides; mPEG-PCL, methoxy poly(ethylene glycol)-block-(ع-caprolactone); PDI, polydispersity index. 
A

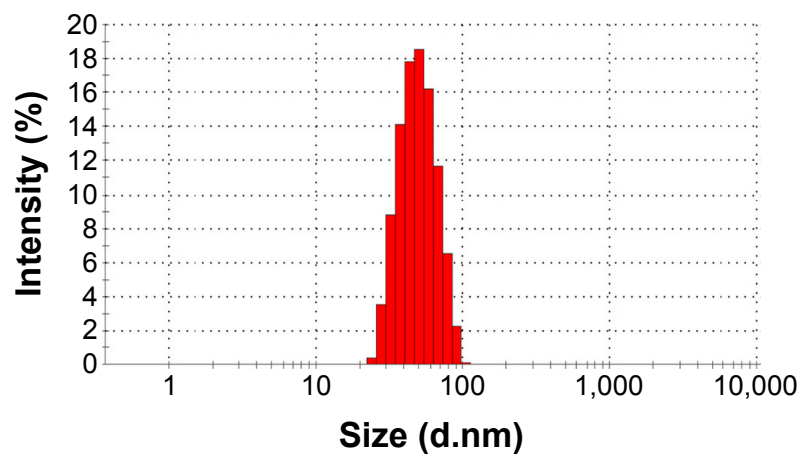

B

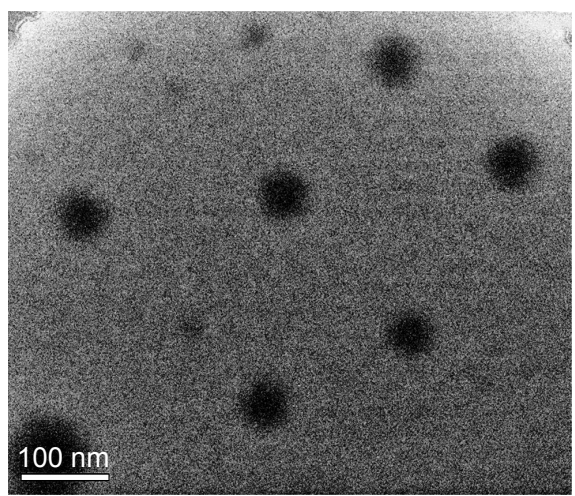

Figure 5 Characterization of Gen-MEs.

Notes: (A) Particle size distribution; (B) morphology of Gen-MEs observed by TEM.

Abbreviations: Gen-MEs, genistein-loaded micellar emulsions; TEM, transmission electron microscopy.

large and tended to precipitate drug due to supersaturation. A low organic/aqueous phase ratio was favorable to MEs assembly and keep them stable. Thus, the ratio of ethanol to water was set as 1:5 to produce Gen-MEs.

Taking above together, a final formulation was confirmed that consisted of $40 \mathrm{mg}$ Gen, $450 \mathrm{mg}$ mPEG-PCL, and $50 \mathrm{mg}$ MCT. Gen-MEs prepared from such formulation was $46.1 \mathrm{~nm}$ in particle size with a polydispersity index of 0.087 , and the $\zeta$ potential was $-0.545 \mathrm{mV}$ (Figure $5 \mathrm{~A}$ ). The nature of small size can avoid rapid clearance of nanoparticles from the body and prevent capture by the RES..$^{28}$ Of note, there were almost no changes in particle size and $\zeta$ potential of GenMEs when compared with the blank carriers. This indicated that the devised MEs were rather suitable for Gen loading. Figure 5B shows that MEs were spherical in morphology as observed by TEM. The EE and DL of MEs toward Gen were $92.6 \%$ and $5.1 \%$, respectively. The concentration of Gen in MEs could be up to $4.0 \mathrm{mg} / \mathrm{mL}$, a value satisfactory for intravenous administration.

\section{In vitro release}

Figure 6 shows the release profiles of Gen from Gen-MEs and Gen-micelles. The release of Gen increased as a function of time, indicating that Gen could be continuously released into the dialysis medium. In the first 12 hours, the releases of Gen from two carriers were fast and similar in kinetics. After that, it could be observed that a slower release proceeded for MEs formulation, which was distinct from the micelles. At 48 hours, the accumulative release of Gen-micelles was $17.7 \%$ higher than that of Gen-MEs. The difference in drug release could be attributed to the disparity of Gen appetence with carrier materials. The release profiles of two preparations were fitted using various model equations and best conformed to the first kinetic process. From the release kinetics, it could be speculated that the major portion of drug would be retained in carriers in the first several hours following administration. A slow release also imparted MEs a long circulation lifetime relative to the micelle carriers. From this point, MEs as injectable carriers of Gen were superior to the micelles, which may be influential in its tissue distribution.

\section{Physical and physiological stability}

Stability is an important parameter for formulation that determines the reproducibility of function and the safety of use. The particle size changes of Gen-MEs and Genmicelles as a function of time are presented in Figure 7. It was showed that two nanocarriers varied little in particle size and polydispersity index at $4^{\circ} \mathrm{C}$ (Figure 7A), demonstrating an excellent physical stability at low temperature. However, at room temperature, the change of

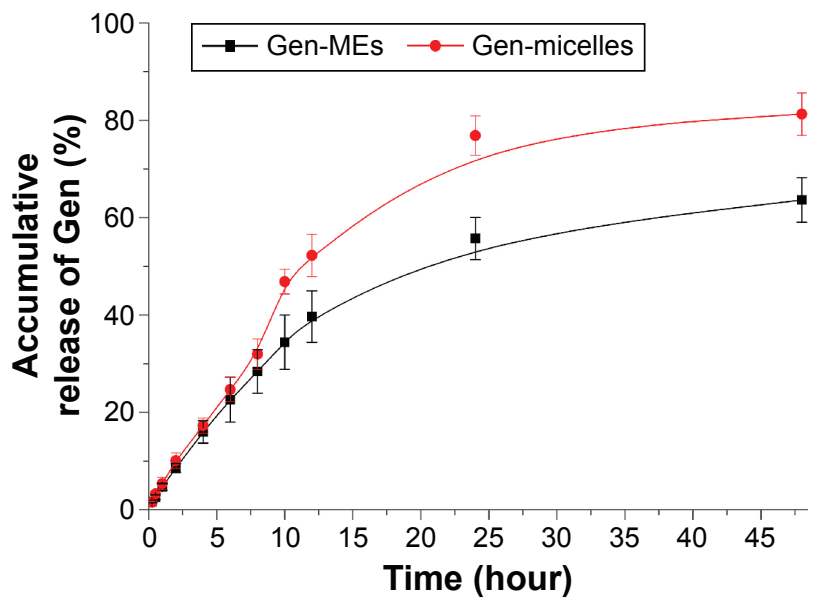

Figure 6 Release curves of Gen-MEs and Gen-micelles performed in $\mathrm{pH} 7.4$ medium based on an equilibrium dialysis method $(n=3$, mean $\pm S D)$.

Abbreviations: Gen, genistein; Gen-MEs, Gen-loaded micellar emulsions. 
A

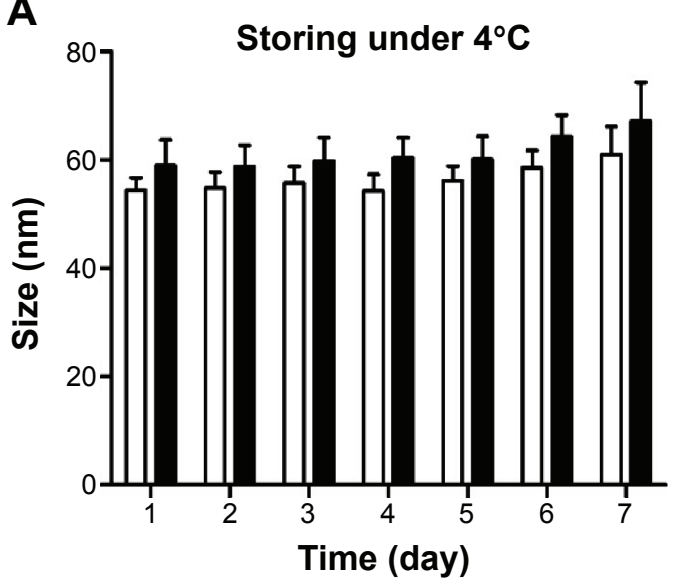

C

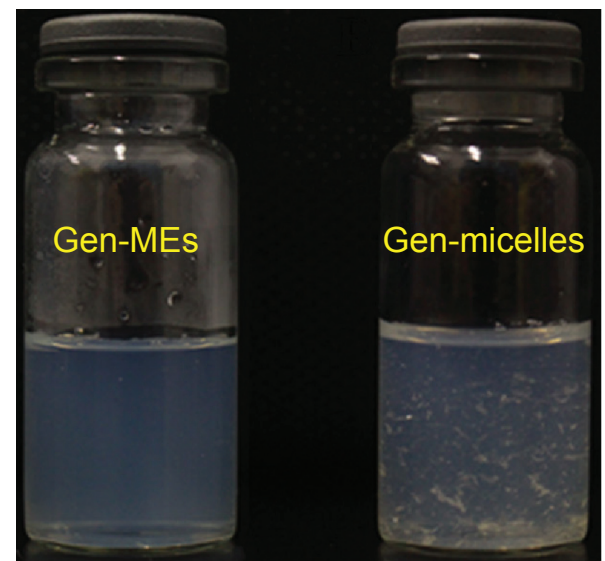

B
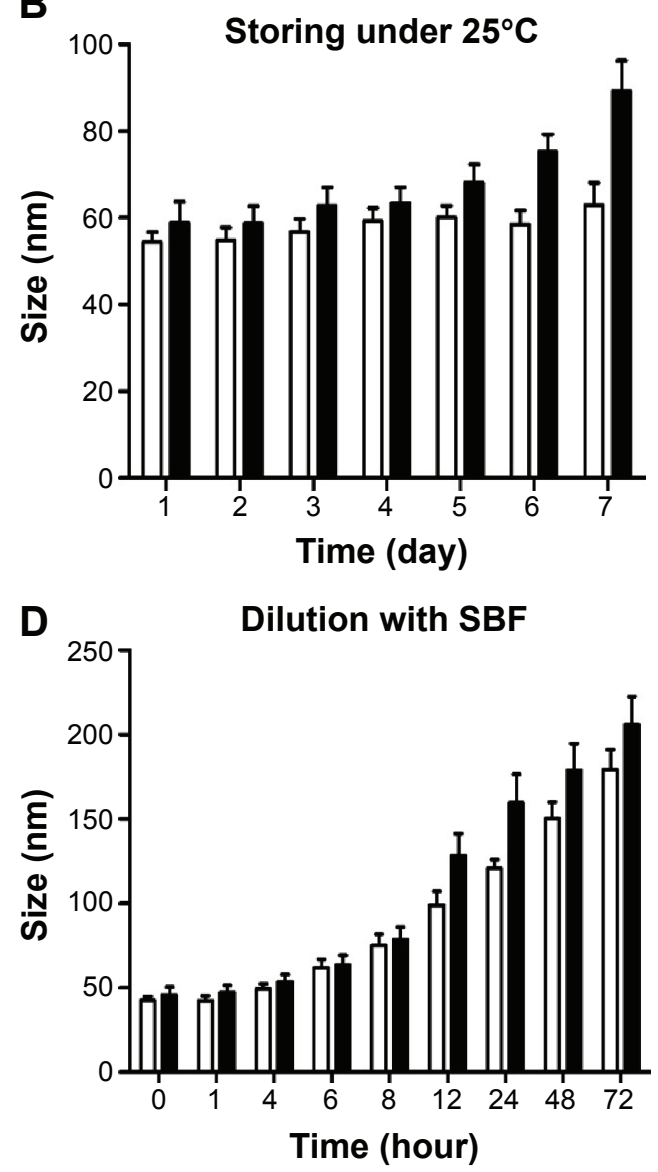

Gen-MEs Gen-micelles

Figure 7 The stability of Gen-MEs characterized by particle size distribution.

Notes: A short-term (I week) stability investigation on storage performed at $4^{\circ} \mathrm{C}(\mathbf{A})$ and $25^{\circ} \mathrm{C}(\mathbf{B})$; physical changes in appearance for Gen-MEs and Gen-micelles after I week of preparation (C); the physiological stability in the blood estimated with pH 7.4 SBF (D).

Abbreviations: Gen, genistein; Gen-MEs, Gen-loaded micellar emulsions; SBF, simulated body fluid.

particle size for Gen-micelles was evident in comparison with that of Gen-MEs (Figure 7B), which may be caused by aggregation of micelles or growth of micellar cores. Besides, drug recrystallization took place in the case of Gen-micelles, accompanying the formation of acicular crystals (Figure 7C), whereas Gen-MEs showed no drug expulsion from MEs. Those results indicated that MEs possessed a better compatibility with Gen and inhibitory effect on recrystallization than micelles.

When injected into the body, the nanosuspensions will be largely diluted by the blood stream that possibly results in dissociation or damage of carriers. Insights into the behavior of nanocarriers in the physiological fluids are highly helpful to understand the biofate of them. The particle size changes of Gen-MEs and Gen-micelles upon circulation in an excess of SBF are shown in Figure 7D. The particle size of two formulations gradually increased with the time as circulating in the SBF. The particle size of Gen-MEs increased from 46.1 to $97.4 \mathrm{~nm}$, whereas it changed from 48.2 to $124.8 \mathrm{~nm}$ for Gen-micelles within 12 hours. In terms of Gen-micelles, the change in particle size was more evident after 12 hours, indicating that MEs had an improved stability against dilution relative to Gen-micelles. This can be ascribable to the reinforced cohesion of hydrophobic core due to the recruitment of MCT that enhanced the robustness of the core via hydrophobic interaction. ${ }^{29}$ The increase of mean particle size for micelle system was speculated from the amalgamation of small particles as a result of Ostwald ripening and aggregation after disassembly of micelles. ${ }^{30}$ This phenomenon could also be explained by apparent Gen precipitation due to thermodynamic instability. Therefore, MEs can be regarded as a betterment of micellar carriers by which the drug loading and physiological stability are expected ameliorative. 


\section{Improved pharmacokinetics}

The plasma drug concentration vs time curves after intravenous administration of Gen-MEs, Gen-micelles, and Gen solution are shown in Figure 8. Pharmacokinetic parameters of Gen are extracted by WinNonlin software using a twocompartment model and listed in Table 1. The blood drug concentrations of Gen-MEs were always higher than those of Gen-micelles and Gen solution. The elimination half-life $\left(t_{1 / 2 \beta}\right)$ of Gen was notably extended through using MEs or micelles formulation compared with Gen solution. In the case of Gen-MEs, the $\mathrm{AUC}_{0-t}$ was $8.48 \mu \mathrm{M} *$ hour that was 1.52- and 2.86-fold as much as that of Gen-micelles and Gen solution, respectively. The mean retention time (MRT) of Gen-MEs was also significantly higher than that of Genmicelles and Gen solution, respectively, 1.57- and 7.38-fold of them. In contrast, the clearance (CL) for Gen-MEs group was significantly lower than that of Gen-micelles and Gen solution, especially in the periphery compartment (as signified by $\mathrm{CL}_{2}$ ), indicating a longer circulation half-life hold by MEs. The pharmacokinetic results demonstrated that MEs are endued with improved systemic circulation and reduced blood elimination in contrast with micelles. The amelioration in pharmacokinetics can be attributed to the contribution of MCT that strengthens the hydrophobic core and lowers the probability of disassembly of nanocarriers. In this study, MEs were originally explored for systemic delivery of Gen, possibly also suitable for other anticancer drugs.

\section{Tissue distribution}

Tissue distribution of drug determinates the drug concentration in the target site and has profound effect on therapeutic prevention and toxicology. Gen levels in the tissues of heart, liver, spleen, lung, kidney, and intestine, delivered with

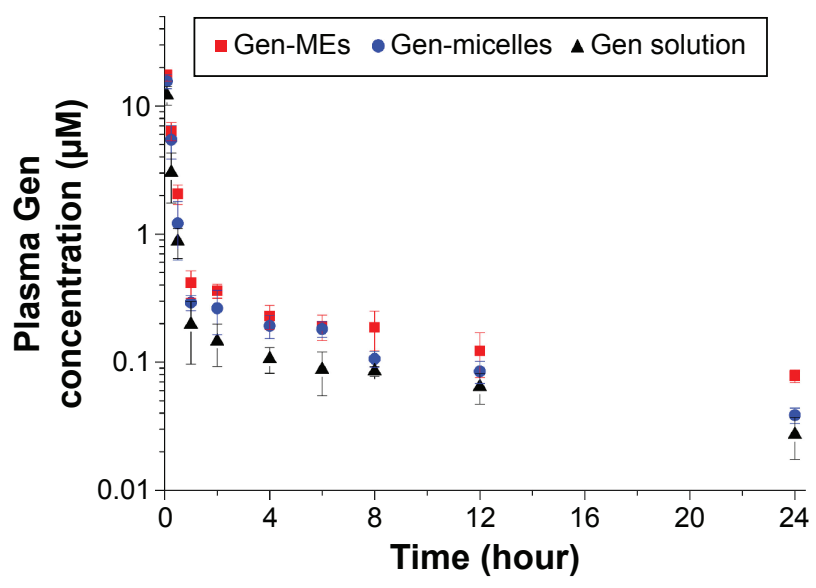

Figure 8 Plasma Gen concentration vs time profiles after intravenous administration of Gen-MEs, Gen-micelles, and Gen solution to rats (mean $\pm S D, n=5$ ).

Abbreviations: Gen, genistein; Gen-MEs, Gen-loaded micellar emulsions.
Table I List of pharmacokinetic parameters derived by fitting the conventional two-compartment model to the plasma data

\begin{tabular}{|c|c|c|c|c|}
\hline Parameter & Unit & Gen-MEs & Gen-micelles & Gen solution \\
\hline$k_{10}$ & $\mathrm{I} / \mathrm{h}$ & $3.22 \pm 1.27^{* *}$ & $4.6 \mathrm{I} \pm 1.54^{* *}$ & $7.68 \pm 2.32$ \\
\hline$t_{1 / 2 \alpha}$ & $\mathrm{h}$ & $0.10 \pm 0.04$ & $0.11 \pm 0.03$ & $0.07 \pm 0.03$ \\
\hline$t_{1 / 2 \beta}$ & $\mathrm{h}$ & $9.96 \pm 3.27 * *$ & $7.5 \pm 3.39 *$ & $2.15 \pm 2.35$ \\
\hline$C L$ & $\mathrm{~L} / \mathrm{h}$ & $1.28 \pm 0.37 * *$ & $1.33 \pm 0.36 * *$ & $2.57 \pm 0.43$ \\
\hline $\mathrm{CL}_{2}$ & $\mathrm{~L} / \mathrm{h}$ & $0.73 \pm 0.21 * * \ldots \#$ & $1.53 \pm 0.15^{* *}$ & $2.94 \pm 0.29$ \\
\hline$A \cup C_{0-t}$ & $\mu M^{*} \mathrm{~h}$ & $8.48 \pm 1.33 * * \ldots$ & $5.55 \pm 0.59 * *$ & $2.97 \pm 0.73$ \\
\hline$A \cup C_{0-\text { inf }}$ & $\mu M *_{h}$ & $9.44 \pm 1.93^{* *, \#}$ & $6.52 \pm 1.53 * *$ & $3.99 \pm 0.77$ \\
\hline AUMC & $\mu M^{*} h^{2}$ & $38.27 \pm 5.21 * * \ldots$ & $27.93 \pm 3.99 * *$ & $5.14 \pm 1.63$ \\
\hline MRT & $\mathrm{h}$ & $8.27 \pm 1.28^{* *, \#}$ & $5.27 \pm 1.56 * *$ & $1.12 \pm 1.26$ \\
\hline$V_{s s}$ & $\mathrm{~L}$ & $6.98 \pm 1.51 * *$ & $7.03 \pm 3.11^{*}$ & $2.52 \pm 2.4 \mathrm{I}$ \\
\hline
\end{tabular}

Notes: Student's t-test, ${ }^{*} P<0.05,{ }^{*} * P<0.01$, compared with Gen solution; ${ }^{*} P<0.05$, ${ }^{\#} P<0.01$, compared with Gen-micelles. Data are presented as mean \pm standard deviation.

Abbreviations: Gen, genistein; Gen-MEs, Gen-loaded micellar emulsions; h, hour; $k_{10}$, elimination rate constant from the central compartment; $t_{1 / 20}$, redistribution halflife; $t_{1 / 22}$, elimination half-life; $C L$, clearance of central compartment; $\mathrm{CL}_{2}$, clearance of peripheral compartment; AUC, area under the plasma concentration-time curve; AUMC, area under the first moment of the plasma concentration-time curve; MRT, mean retention time; $V_{\mathrm{ss}}$, steady-state volume of distribution.

the form of MEs and micelles, are presented in Figure 9. It could be observed that Gen was promptly distributed into the selective tissues after administration and followed a varied decline in different organs. In the heart, the drug distribution was lowest and dropped slowly with the time (Figure 9A), indicating that nanocarriers with PEG shell can reduce the drug perfusion into the heart, and thus reduce the cardiac toxicity. However, there was no significant difference between MEs and micelles. In the organs of liver, spleen, lung, and kidney (Figure 9B-E), rapid attenuation in drug distribution happened after 2 hours. As an exception, the Gen distribution in the intestine was remarkably higher than in other tissues and it actually raised with the time (Figure 9F). It was indicative that an intestinal secretion likely involved in the disposition of Gen, which may be potential to topically treat the intestinal cancers.

As for the drug distribution between Gen-MEs and Gen-micelles, it was also significantly different in the liver, kidney, and intestine. As known, the liver and kidney are the vital organs responsible for drug metabolism and elimination. Micelles are easily sequestered into the RES, such as the liver, spleen, and lymphatic tissues. ${ }^{31}$ Dissociation of micelles under the action of blood stream accelerated the process of drug distribution into the organs in charge of drug elimination. Higher accumulation in the liver and kidney represented a rapid elimination from the body. It was clear that Gen-MEs showed less distribution in the liver and kidney than Gen-micelles at 2 hours, a key time point for distribution, which in turn elongated the elimination half-life. This was also consistent with the result of pharmacokinetics. 

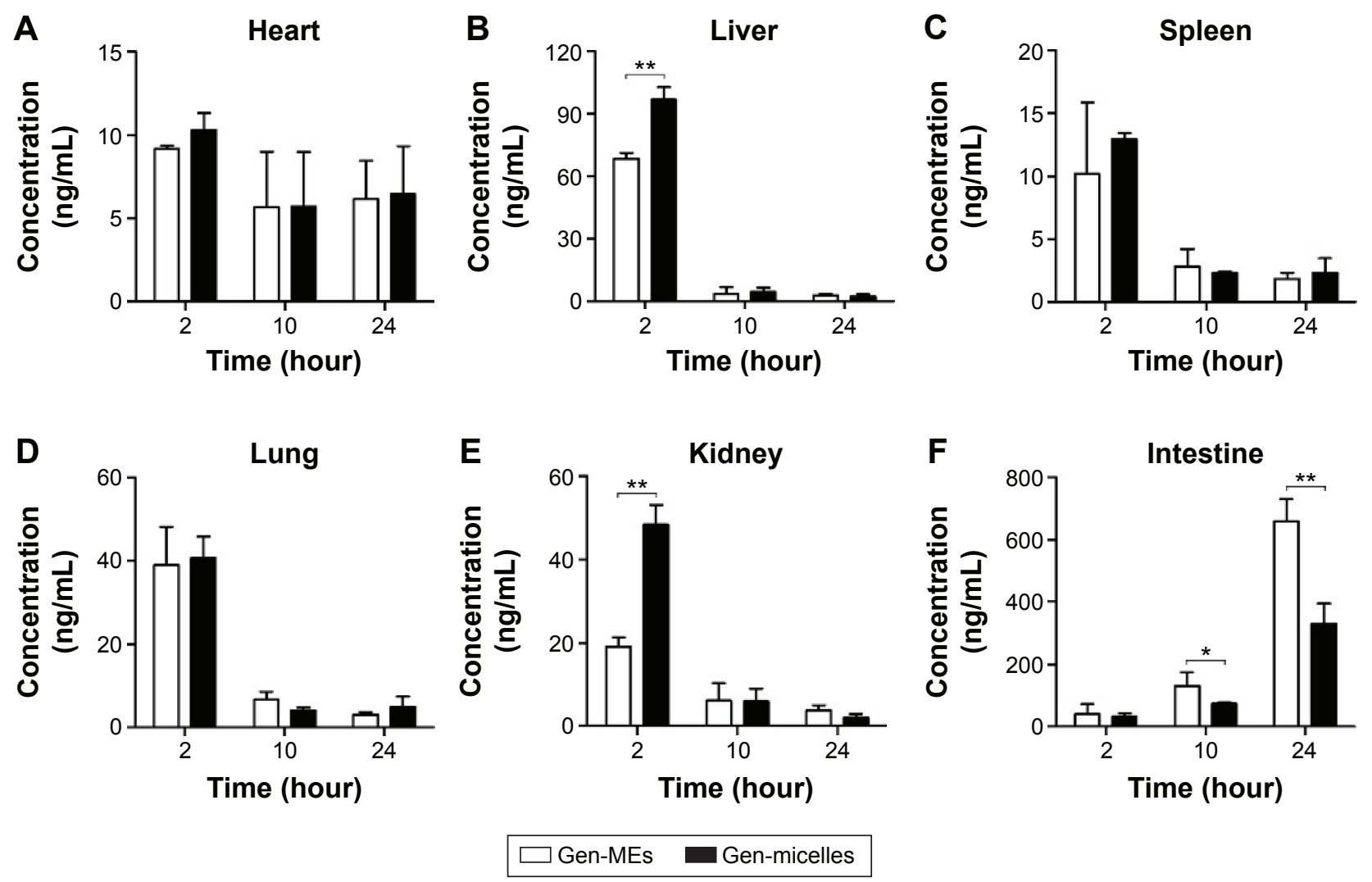

Figure 9 Tissue distribution of Gen after intravenous administration of Gen-MEs and Gen-micelles at 2, 10, and 24 hours. Notes: Student's $t$-test, $* * P<0.01$, compared with Gen-micelles.

Abbreviations: Gen, genistein; Gen-MEs, Gen-loaded micellar emulsions.

The research on tissue distribution has lent a strong support that MEs possess a better physiological stability than micelles as circulating in the body.

\section{Conclusion}

This study demonstrated the suitability of MEs as an alternative of micelles for systemic delivery of Gen. MEs were specially designed based on micelles whereby to improve the stability of carriers and the tissue distribution of delivered drug. The nanocarriers of MEs possessed better physical and physiological stability compared with micelles. Pharmacokinetic study showed that MEs could significantly prolong the blood circulation half-life and enhance the AUC of drug. The results of tissue distribution indicated that MEs could make the payload less perfusion into the liver and kidney relative to micelles. Taken together, MEs have been proved suitable for systemic delivery of Gen, which may be promising as novel nanocarriers of poorly soluble drugs for improvement of pharmacokinetics.

\section{Acknowledgment}

This work was supported by the National Natural Science Foundation of China (81402855).

\section{Disclosure}

The authors report no conflicts of interest in this work.

\section{References}

1. Li HF, Wang LD, Qu SY. Phytoestrogen genistein decreases contractile response of aortic artery in vitro and arterial blood pressure in vivo. Acta Pharmacol Sin. 2004;25(3):313-318.

2. Taylor CK, Levy RM, Elliott JC, Burnett BP. The effect of genistein aglycone on cancer and cancer risk: a review of in vitro, preclinical, and clinical studies. Nutr Rev. 2009;67(7):398-415.

3. Busby MG, Jeffcoat AR, Bloedon LT, et al. Clinical characteristics and pharmacokinetics of purified soy isoflavones: single-dose administration to healthy men. Am J Clin Nutr. 2002;75(1):126-136.

4. Yanagihara N, Zhang H, Toyohira $\mathrm{Y}$, et al. New insights into the pharmacological potential of plant flavonoids in the catecholamine system. J Pharmacol Sci. 2014;124(2):123-128.

5. Miao Q, Li JG, Miao S, et al. The bone-protective effect of genistein in the animal model of bilateral ovariectomy: roles of phytoestrogens and PTH/PTHR1 against post-menopausal osteoporosis. Int J Mol Sci. 2012;13(1):56-70.

6. Sarkar FH, Li Y. The role of isoflavones in cancer chemoprevention. Front Biosci. 2004;9:2714-2724.

7. Bektic J, Guggenberger R, Eder IE, et al. Molecular effects of the isoflavonoid genistein in prostate cancer. Clin Prostate Cancer. 2005; 4(2):124-129.

8. Banerjee S, Li Y, Wang Z, Sarkar FH. Multi-targeted therapy of cancer by genistein. Cancer Lett. 2008;269(2):226-242.

9. Spinozzi F, Pagliacci MC, Migliorati G, et al. The natural tyrosine kinase inhibitor genistein produces cell cycle arrest and apoptosis in Jurkat T-leukemia cells. Leuk Res. 1994;18(6):431-439. 
10. Prietsch RF, Monte LG, da Silva FA, et al. Genistein induces apoptosis and autophagy in human breast MCF-7 cells by modulating the expression of proapoptotic factors and oxidative stress enzymes. $\mathrm{Mol}$ Cell Biochem. 2014;390(1-2):235-242.

11. Liu YL, Zhang GQ, Yang Y, Zhang CY, Fu RX, Yang YM. Genistein induces G2/M arrest in gastric cancer cells by increasing the tumor suppressor PTEN expression. Nutr Cancer. 2013;65(7):1034-1041.

12. Wu JG, Ge JA, Zhang YP, Yu Y, Zhang XY. Solubility of genistein in water, methanol, ethanol, propan-2-ol, 1-butanol, and ethyl acetate from (280 to 333) K. J Chem Eng Data. 2010;55(11):5286-5288.

13. Mattheolabakis G, Nie T, Constantinides PP, Rigas B. Sterically stabilized liposomes incorporating the novel anticancer agent phosphoibuprofen (MDC-917): preparation, characterization, and in vitro/ in vivo evaluation. Pharm Res. 2012;29(6):1435-1443.

14. Zhang Y, Zhang X, Liu H, Cai S, Wu B. Mixed nanomicelles as potential carriers for systemic delivery of Z-GP-Dox, an FAPalpha-based doxorubicin prodrug: formulation and pharmacokinetic evaluation. Int J Nanomed. 2015;10:1625-1636.

15. Ganta S, Talekar M, Singh A, Coleman TP, Amiji MM. Nanoemulsions in translational research-opportunities and challenges in targeted cancer therapy. AAPS PharmSciTech. 2014;15(3):694-708.

16. Madan J, Pandey RS, Jain V, Katare OP, Chandra R, Katyal A. Poly (ethylene)-glycol conjugated solid lipid nanoparticles of noscapine improve biological half-life, brain delivery and efficacy in glioblastoma cells. Nanomedicine. 2013;9(4):492-503.

17. Zheng J, Wan Y, Elhissi A, Zhang Z, Sun X. Targeted paclitaxel delivery to tumors using cleavable PEG-conjugated solid lipid nanoparticles. Pharm Res. 2014;31(8):2220-2233.

18. Jiang X, Xin H, Ren Q, et al. Nanoparticles of 2-deoxy-D-glucose functionalized poly(ethylene glycol)-co-poly(trimethylene carbonate) for dual-targeted drug delivery in glioma treatment. Biomaterials. 2014;35(1):518-529.

19. Zhou Z, Badkas A, Stevenson M, Lee JY, Leung YK. Herceptin conjugated PLGA-PHis-PEG pH sensitive nanoparticles for targeted and controlled drug delivery. Int J Pharm. 2015;487(1-2):81-90.

20. Mohamed S, Parayath NN, Taurin S, Greish K. Polymeric nanomicelles: versatile platform for targeted delivery in cancer. Ther Deliv. 2014;5(10):1101-1121.
21. Zhang X, Wang H, Ma Z, Wu B. Effects of pharmaceutical PEGylation on drug metabolism and its clinical concerns. Expert Opin Drug Metab Toxicol. 2014;10(12):1691-1702.

22. Shiraishi K, Hamano M, Ma H, et al. Hydrophobic blocks of PEGconjugates play a significant role in the accelerated blood clearance (ABC) phenomenon. J Control Release. 2013;165(3):183-190.

23. Zhang $\mathrm{X}$, Wang $\mathrm{H}$, Zhang $\mathrm{T}$, Zhou X, Wu B. Exploring the potential of self-assembled mixed micelles in enhancing the stability and oral bioavailability of an acid-labile drug. Eur J Pharm Sci. 2014; 62:301-308.

24. Zhou Q, Mu K, Jiang L, et al. Glioma-targeting micelles for optical/ magnetic resonance dual-mode imaging. Int J Nanomed. 2015;10: $1805-1818$

25. Kokubo T, Takadama H. How useful is SBF in predicting in vivo bone bioactivity? Biomaterials. 2006;27(15):2907-2915.

26. Zhang X, Zhang T, Zhou X, et al. Enhancement of oral bioavailability of tripterine through lipid nanospheres: preparation, characterization, and absorption evaluation. J Pharm Sci. 2014;103(6):1711-1719.

27. Liu W, Liu H, Sun H, et al. Metabolite elucidation of the Hsp90 inhibitor SNX-2112 using ultraperformance liquid chromatography/quadrupole time-of-flight mass spectrometry (UPLC-QTOF/MS). Xenobiotica. 2014;44(5):455-464.

28. Wang X, Wang Y, Chen ZG, Shin DM. Advances of cancer therapy by nanotechnology. Cancer Res Treat. 2009;41(1):1-11.

29. Benson SP, Pleiss J. Molecular dynamics simulations of selfemulsifying drug-delivery systems (SEDDS): influence of excipients on droplet nanostructure and drug localization. Langmuir. 2014; 30(28):8471-8480.

30. Bayati S, Zhu K, Trinh LT, Kjoniksen AL, Nystrom B. Effects of temperature and salt addition on the association behavior of charged amphiphilic diblock copolymers in aqueous solution. J Phys Chem B. 2012;116(36):11386-11395.

31. Kwon GS, Kataoka K. Block copolymer micelles as long-circulating drug vehicles. Adv Drug Del Rev. 2012;64:237-245.
International Journal of Nanomedicine

\section{Publish your work in this journal}

The International Journal of Nanomedicine is an international, peerreviewed journal focusing on the application of nanotechnology in diagnostics, therapeutics, and drug delivery systems throughout the biomedical field. This journal is indexed on PubMed Central, MedLine, CAS, SciSearch ${ }^{\circledR}$, Current Contents ${ }^{\circledR} /$ Clinical Medicine,

\section{Dovepress}

Journal Citation Reports/Science Edition, EMBase, Scopus and the Elsevier Bibliographic databases. The manuscript management system is completely online and includes a very quick and fair peer-review system, which is all easy to use. Visit http://www.dovepress.com/ testimonials.php to read real quotes from published authors. 